\title{
On multiple higher Mahler measures and Witten zeta values associated with semisimple Lie algebras
}

\author{
YOSHITAKA SASAKI
}

\begin{abstract}
The Witten zeta-functions associated with semisimple Lie algebras were defined by Zagier, and their special values at even positive integers were first studied by Witten in connection with quantum gauge theory. In this paper, relations between multiple higher Mahler measures for some families of polynomials and special values of Witten zeta-functions at positive integers are showed. Consequently, a geometrical interpretation of the multiple higher Mahler measure as the volume of certain moduli space is given.
\end{abstract}

\section{Introduction}

The Mahler measure of a Laurent polynomial $P \in \mathbb{C}\left[X_{1}^{ \pm 1}, \ldots, X_{n}^{ \pm 1}\right] \backslash\{0\}$ is defined by

$$
\mathrm{m}(P):=\int_{0}^{1} \cdots \int_{0}^{1} \log \left|P\left(\mathrm{e}^{2 \pi \mathrm{i} t_{1}}, \ldots, \mathrm{e}^{2 \pi \mathrm{i} t_{n}}\right)\right| d t_{1} \cdots d t_{n} .
$$

Various properties of the Mahler measure, for instance, relationships with special values of $L$-functions, the volumes of certain manifolds and so on, have been discovered.

Recently, the multiple higher Mahler measure was introduced by Kurokawa et al. [6] as a generalization of the Mahler measure.

Definition 1.1 The multiple higher Mahler measure [6]. Let $\mathscr{P}:=$ $\left\{P_{j}\right\}_{j=1}^{l}$ be a family of Laurent polynomials $P_{j} \in \mathbb{C}\left[X_{1}^{ \pm 1}, \ldots, X_{n}^{ \pm 1}\right] \backslash\{0\}$. Then the multiple higher Mahler measure of $\mathscr{P}$ is defined by

$\mathrm{m}(\mathscr{P})=\mathrm{m}\left(P_{1}, \ldots, P_{l}\right):=\int_{0}^{1} \cdots \int_{0}^{1} \prod_{j=1}^{l} \log \left|P_{j}\left(\mathrm{e}^{2 \pi \mathrm{i} t_{1}}, \ldots, \mathrm{e}^{2 \pi \mathrm{i} t_{n}}\right)\right| d t_{1} \cdots d t_{n}$ 
In [6], many examples of multiple higher Mahler measures for some families of polynomials were calculated. For instance, they gave the following formula:

$$
\begin{gathered}
\mathrm{m}\left(1-x, 1-\mathrm{e}^{2 \pi \mathrm{i} \alpha} x, 1-\mathrm{e}^{2 \pi i \beta} x\right)=-\frac{1}{4}\left(\sum_{k=1}^{\infty} \sum_{l=1}^{\infty} \frac{\cos (2 \pi((k+l) \beta-l \alpha))}{k l(k+l)}\right. \\
\left.+\sum_{k=1}^{\infty} \sum_{l=1}^{\infty} \frac{\cos (2 \pi((k+l) \alpha-l \beta))}{k l(k+l)}+\sum_{k=1}^{\infty} \sum_{l=1}^{\infty} \frac{\cos (2 \pi(l \alpha+k \beta))}{k l(k+l)}\right) .
\end{gathered}
$$

The double series appearing in the right-hand side of the above formula are a kind of Tornheim's double series. Tornheim's double series is also defined as the special value of the multi-variable Witten zeta-function associated with $\mathfrak{s l}(3)$ (or $A_{2}$, see (5.1) below).

In this paper, we first show relations between multiple higher Mahler measures for some families of polynomials and special values of the multivariable Witten zeta-functions for semisimple Lie algebras at positive integers. Consequently, a geometrical interpretation of the multiple higher Mahler measure as the volume of certain moduli space is naturally derived from the property of the Witten zeta-function.

The Witten zeta-function associated with a semisimple Lie algebra $\mathfrak{g}$ is defined by

$$
\zeta_{W}(s ; \mathfrak{g}):=\sum_{\varphi}(\operatorname{dim} \varphi)^{-s}
$$

where $\varphi$ runs over all finite dimension irreducible representations of $\mathfrak{g}$. The above definition is due to Zagier [16], and the values $\zeta_{W}(2 k ; \mathfrak{g})$ for positive integers $k$ were first studied by Witten [17] in order to express the volumes of the moduli spaces of flat connections on $G$ bundles over the compact 2-manifold, where $G$ is the semisimple compact Lie group. Therefore, as mentioned above, our main theorem indicates that multiple higher Mahler measures for some families of polynomials can be interpreted as the volumes of such moduli spaces. Zagier noted in [16] that

$$
\zeta_{W}(2 k ; \mathfrak{g}) \in \mathbb{Q} \pi^{k l} \quad(k \in \mathbb{N}),
$$

where $l$ is the number of positive roots of $\mathfrak{g}$, by using Witten's result [17]. This formula is called "Witten's volume formula". Some explicit formulas for $\zeta_{W}(2 k, \mathfrak{g})(k \in \mathbb{N})$ were given by Mordell [9], Zagier [16], Subbarao and Sitaramachandrarao [12] and Gunnells and Sczech [1]. Further Matsumoto 
and Tsumura [8] and Komori et al. [3] introduced the multi-variable Witten zeta-functions associated with semisimple Lie algebras, and evaluated special values at positive integers of those functions, including $\zeta_{W}(2 k ; \mathfrak{g})$, for some $\mathfrak{g}$ explicitly (see $[2,4,5,8]$ ).

In the next section, we describe the definition of the multi-variable Witten zeta-function associated with $\mathfrak{g}$, which is due to Komori, Matsumoto and Tsumura. In Section 3, we introduce some notation and state the main theorem as Theorem 3.2. The proof of Theorem 3.2 is given in Section 4. In the last section, some examples of Theorem 3.2 is presented.

\section{The multi-variable Witten zeta-function}

In this section, we describe the definition of the multi-variable Witten zetafunctions associated with semisimple Lie algebras, which is mentioned in terms of roots and weights for the corresponding root systems by using Weyl's dimension formula. As mentioned above, the following definition is due to Matsumoto and Tsumura [8] and Komori et al. [3]. Matsumoto and Tsumura [8] first introduced the $\mathfrak{s l}(l)$ case. Afterwards, Komori et al. [3] introduced the other cases (also see [2]).

Let $\mathfrak{g}$ be a semisimple Lie algebra of rank $r$. We denote the set of all roots of $\mathfrak{g}$ by $\Delta$, the set of all positive roots (resp. negative roots) by $\Delta_{+}$(resp. $\Delta_{-}$) and the fundamental system of $\Delta$ by $\Pi$. For any $\alpha \in \Delta$, we denote the associated coroot by $\alpha^{\vee}$. Let $\lambda_{\alpha}(\alpha \in \Pi)$ be the fundamental weights satisfying $\left\langle\beta^{\vee}, \lambda_{\alpha}\right\rangle=\delta_{\beta, \alpha}$ (Kronecker's delta) for $\alpha, \beta \in \Pi$. We put

$$
P:=\bigoplus_{\alpha \in \Pi} \mathbb{Z} \lambda_{\alpha} \quad \text { and } \quad P_{++}:=\bigoplus_{\alpha \in \Pi} \mathbb{Z}_{\geq 1} \lambda_{\alpha}
$$

Then we define the multi-variable Witten zeta-function associated with $\mathfrak{g}$ by

$$
\zeta_{r}(\mathbf{s} ; \mathfrak{g}):=\sum_{\lambda \in P_{++}} \prod_{\alpha \in \Delta_{+}}\left\langle\alpha^{\vee}, \lambda\right\rangle^{-s_{\alpha}}
$$

where $\mathbf{s}=\left(s_{\alpha}\right)_{\alpha \in \Delta_{+}} \in \mathbb{C}^{n}\left(n=\left|\Delta_{+}\right|\right)$. The Witten zeta-function (1.1) can be expressed as

$$
\zeta_{W}(s ; \mathfrak{g})=K(\mathfrak{g})^{s} \zeta_{r}(\underbrace{s, \ldots, s}_{n} ; \mathfrak{g})
$$

where $K(\mathfrak{g}):=\prod_{\beta \in \Delta_{+}}\left\langle\beta^{\vee}, \sum_{\alpha \in \Pi} \lambda_{\alpha}\right\rangle$. If $\mathfrak{g}$ is of type $X_{r}(X=A, B, C, D, E$, $F, G)$, then we also denote $\zeta_{r}\left(\mathbf{s} ; X_{r}\right)$ instead of $\zeta_{r}(\mathbf{s} ; \mathfrak{g})$. 


\section{The main theorem}

Hereafter, we assume $\mathbf{s}=\left(s_{\alpha}\right)_{\alpha \in \Delta_{+}}$are positive integers. For $\alpha \in \Delta_{+}$, we put

$$
\begin{aligned}
& X_{\alpha}^{(l)}:=1-x_{\alpha}^{(l)} \quad\left(l=2, \ldots, s_{\alpha}\right), \\
& Z_{\alpha}:=1-\prod_{\beta \in \Delta_{+} \backslash \Pi} z_{\beta}^{C_{\beta, \alpha}} \prod_{l=2}^{s_{\alpha}} x_{\alpha}^{(l)},
\end{aligned}
$$

where $x_{\alpha}^{(l)}$ and $z_{\beta}$ are indeterminate elements and

$$
C_{\beta, \alpha}= \begin{cases}\left\langle\beta^{\vee}, \lambda_{\alpha}\right\rangle & \text { when } \alpha \in \Pi, \\ \delta_{\beta, \alpha} & \text { when } \alpha \in \Delta_{+} \backslash \Pi .\end{cases}
$$

Then we denote the family of those polynomials by

$$
\mathscr{P}(\mathbf{s} ; \mathfrak{g})=\mathscr{P}\left(\mathbf{s} ; X_{r}\right):=\left\{X_{\alpha}^{(l)}, Z_{\alpha} \mid \alpha \in \Delta_{+}, l=2, \ldots, s_{\alpha}\right\}
$$

Remark 3.1. When $s_{\alpha}=1$, we except the corresponding polynomials $X_{\alpha}^{(l)}$ and understand that the corresponding products $\prod_{l=2}^{1} x_{\alpha}^{(l)}$ are equal to 1 .

By using the above notation, we have

Theorem 3.2. For a semisimple Lie algebra $\mathfrak{g}\left(\right.$ type of $\left.X_{r}\right)$ such that $\Delta_{+} \backslash$ $\Pi \neq \emptyset$ and positive integers $\mathbf{s}=\left(s_{\alpha}\right)_{\alpha \in \Delta_{+}}$, we have

$$
\mathrm{m}(\mathscr{P}(\mathbf{s} ; \mathfrak{g}))=\mathrm{m}\left(\mathscr{P}\left(\mathbf{s} ; X_{r}\right)\right)=\frac{(-1)^{S_{n}}}{2^{S_{n}}} \sum_{w \in W} \zeta_{r}(w \mathbf{s} ; \mathfrak{g}),
$$

where $S_{n}:=\sum_{\alpha \in \Pi} s_{\alpha}\left(n=\left|\Delta_{+}\right|\right), W$ is the Weyl group of $X_{r}$ and $w \mathbf{s}$ implies the action of Weyl group to the index defined by $w \mathbf{s}=\left(s_{\left|w^{-1}(\alpha)\right|}\right)_{\alpha \in \Delta_{+}}$.

Remark 3.3. For $\alpha \in \Delta$, we have set $|\alpha|=\alpha$ if $\alpha \in \Delta_{+}$and $|\alpha|=-\alpha$ if $\alpha \in \Delta_{-}$in Theorem 3.2. 


\section{The proof of Theorem 3.2}

We put $\mathbf{e}(\theta):=\mathrm{e}^{2 \pi i \theta}$ and

$$
\mathcal{L}_{k}(\theta):=\sum_{n=1}^{\infty} \frac{\cos (2 \pi n \theta)}{n^{k}} \quad(0<\theta<1, k \geq 1)
$$

Note that $\mathcal{L}_{1}(\theta)=-\log |1-\mathbf{e}(\theta)|$. From the definition of the multiple higher Mahler measure and the above notation, we have

$$
\begin{aligned}
\mathrm{m}(\mathscr{P}(\mathbf{s} ; \mathfrak{g}))= & (-1)^{S_{n}} \int_{0}^{1} \cdots \int_{0}^{1} \prod_{\alpha \in \Delta_{+}} \mathcal{L}_{1}\left(\sum_{l=2}^{s_{\alpha}} t_{\alpha}^{(l)}+\sum_{\beta \in \Delta_{+} \backslash \Pi} C_{\beta, \alpha} v_{\beta}\right) \\
& \times \prod_{l=2}^{s_{\alpha}} \mathcal{L}_{1}\left(t_{\alpha}^{(l)}\right) d \boldsymbol{T} d \boldsymbol{V}
\end{aligned}
$$

where

$$
d \boldsymbol{T} d \boldsymbol{V}=\prod_{\alpha \in \Delta_{+}} \prod_{l=2}^{s_{\alpha}} d t_{\alpha}^{(l)} \prod_{\beta \in \Delta_{+}} d v_{\beta}
$$

and we have put $x_{\alpha}^{(l)}=\mathbf{e}\left(t_{\alpha}^{(l)}\right)$ and $z_{\beta}=\mathbf{e}\left(v_{\beta}\right)$. The change of the order of integration and summation can be justified by the following way: for a given positive integer $\lambda \geq 2$, there exists $\delta(=\delta(\lambda))$ such that

$$
|\log (2-2 \cos (2 \pi x))|<x^{-1 / \lambda}
$$

for any $x \in(0, \delta)$. Therefore, for any $x \in(0, \delta)$ and sufficiently large $R$, we see that

$$
\left|\sum_{l=1}^{R} \frac{\cos (2 \pi l x)}{l}\right|<|\log (2-2 \cos (2 \pi x))|+1<x^{-1 / \lambda}+1 .
$$

From the symmetric property, the left-hand side of the above inequality is estimated by $(1-x)^{-1 / \lambda}+1$ for any $x \in(1-\delta, 1)$. Needless to say, the lefthand side of the above inequality is bounded for any $x \in[\delta, 1-\delta]$. Hence we see that

$$
\int_{0}^{1}\left|\sum_{l=1}^{R} \frac{\cos (2 \pi l(x+\theta))}{l} \sum_{m=1}^{R} \frac{\cos (2 \pi m x)}{m}\right| d x<\infty,
$$


for any $\theta \in[0,1]$. Therefore, by Lebesgue's convergence theorem, we may integrate term-by-term and obtain

$$
\begin{aligned}
\int_{0}^{1} & \sum_{l=1}^{\infty} \frac{\cos (2 \pi l(x+\theta))}{l} \sum_{m=1}^{\infty} \frac{\cos (2 \pi m x)}{m} d x \\
& =\int_{0}^{1} \lim _{R \rightarrow \infty} \sum_{l=1}^{R} \frac{\cos (2 \pi l(x+\theta))}{l} \sum_{m=1}^{R} \frac{\cos (2 \pi m x)}{m} d x \\
& =\lim _{R \rightarrow \infty} \sum_{l=1}^{R} \sum_{m=1}^{R} \frac{1}{l m} \int_{0}^{1} \cos (2 \pi l(x+\theta)) \cos (2 \pi m x) d x=\frac{1}{2} \sum_{l=1}^{\infty} \frac{\cos (2 \pi l \theta)}{l^{2}}
\end{aligned}
$$

Here, we have used

$$
\int_{0}^{1} \cos (2 \pi((l-m) x+\theta)) d x= \begin{cases}\cos 2 \pi \theta & \text { if } l=m \\ 0 & \text { otherwise }\end{cases}
$$

Repeating this manner, we have

$$
\mathrm{m}(\mathscr{P}(\mathbf{s} ; \mathfrak{g}))=\frac{(-1)^{S_{n}}}{2^{S_{n}-n}} \int_{0}^{1} \cdots \int_{0}^{1} \prod_{\alpha \in \Delta_{+}} \mathcal{L}_{S_{\alpha}}\left(\sum_{\beta \in \Delta_{+} \backslash \Pi} C_{\beta, \alpha} v_{\beta}\right) d \boldsymbol{V}
$$

From the definition of $C_{\beta, \alpha}$, the integrand of the above formula can be rewritten as

$$
\prod_{\alpha \in \Pi} \mathcal{L}_{s_{\alpha}}\left(\sum_{\beta \in \Delta_{+} \backslash \Pi}\left\langle\beta^{\vee}, \lambda_{\alpha}\right\rangle v_{\beta}\right) \prod_{\beta \in \Delta_{+} \backslash \Pi} \mathcal{L}_{s_{\alpha}}\left(v_{\beta}\right)
$$

Therefore, performing the integration in (4.4), we have

$$
\begin{aligned}
\operatorname{m}(\mathscr{P}(\mathbf{s} ; \mathfrak{g}))= & \frac{(-1)^{S_{n}}}{2^{S_{n}}} \sum_{\boldsymbol{\sigma} \in \mathfrak{S}} \sum_{\begin{array}{c}
m_{\alpha}>0 \\
\sigma_{\beta}\left\langle\beta^{\vee}, \sum_{\alpha \in \Pi} \sigma_{\alpha} m_{\alpha} \lambda_{\alpha}\right\rangle>0 \\
\text { for } \forall \beta \in \Delta_{+} \backslash \Pi
\end{array}} \prod_{\alpha \in \Pi} \frac{1}{m_{\alpha}^{s_{\alpha}}} \\
& \times \prod_{\beta \in \Delta_{+} \backslash \Pi} \frac{1}{\left(\sigma_{\beta}\left\langle\beta^{\vee}, \sum_{\alpha \in \Pi} \sigma_{\alpha} m_{\alpha} \lambda_{\alpha}\right\rangle\right)^{s_{\beta}}}
\end{aligned}
$$




$$
\begin{aligned}
& =\frac{(-1)^{S_{n}}}{2^{S_{n}}} \sum_{\boldsymbol{\sigma} \in \mathfrak{S}} \sum_{\substack{\sigma_{\alpha} m_{\alpha}>0 \\
\sigma_{\beta}\left\langle\beta^{\vee}, \sum_{\alpha \in \Pi} m_{\alpha} \lambda_{\alpha}\right\rangle>0 \\
\text { for }{ }^{\forall} \in \Delta_{+} \backslash \Pi}} \cdots \prod_{\alpha \in \Pi} \frac{1}{\left|m_{\alpha}\right|^{s_{\alpha}}} \\
& \quad \times \prod_{\beta \in \Delta_{+} \backslash \Pi} \frac{1}{\left|\left\langle\beta^{\vee}, \sum_{\alpha \in \Pi} m_{\alpha} \lambda_{\alpha}\right\rangle\right|^{s_{\beta}}} .
\end{aligned}
$$

Here we have put $\boldsymbol{\sigma}=\left(\left(\sigma_{\alpha}\right)_{\alpha \in \Delta_{+}}\right) \in \mathfrak{S}:=\{ \pm 1\}^{n}$. By performing the summation for $\boldsymbol{\sigma} \in \mathfrak{S}$, the inner series in the above formula can be rewritten as follows:

$$
\begin{aligned}
\mathrm{m}(\mathscr{P}(\mathbf{s} ; \mathfrak{g}))= & \frac{(-1)^{S_{n}}}{2^{S_{n}}} \sum_{\substack{m_{\alpha} \neq 0 \\
\left\langle\beta^{\vee}, \sum_{\alpha \in \Pi} m_{\alpha} \lambda_{\alpha}\right\rangle \neq 0 \\
\text { for } \forall \in \Delta_{+} \backslash \Pi}} \cdots \prod_{\alpha \in \Pi} \frac{1}{\left|m_{\alpha}\right|^{s_{\alpha}}} \\
& \times \prod_{\substack{\beta \in \Delta_{+} \backslash \Pi\\
}} \frac{1}{\left|\left\langle\beta^{\vee}, \sum_{\alpha \in \Pi} m_{\alpha} \lambda_{\alpha}\right\rangle\right|^{s_{\beta}}} \\
= & \frac{(-1)^{S_{n}}}{2^{S_{n}}} \sum_{\substack{\lambda \in P \\
\left\langle\alpha^{\vee}, \lambda\right\rangle \neq 0 \text { for }{ }^{\forall} \alpha \in \Delta_{+}}} \prod_{\alpha \in \Delta_{+}} \frac{1}{\left|\left\langle\alpha^{\vee}, \lambda\right\rangle\right|^{s_{\alpha}}} .
\end{aligned}
$$

The last formula implies that the sum of Witten zeta values for the action of the corresponding Weyl group. Thus, we have Theorem 3.2.

\section{Examples}

\section{1. $A_{2}$ case}

The multi-variable Witten zeta-function associated with $A_{2}(\mathfrak{s l}(3))$ is

$$
\zeta_{2}\left(s_{1}, s_{2}, s_{3} ; A_{2}\right)=\sum_{m, n=1}^{\infty} \frac{1}{m^{s_{1}} n^{s_{2}}(m+n)^{s_{3}}} .
$$

The above series was already introduced by Tornheim [13] in 1950. Therefore, (5.1) is so-called "Tornheim's double series". Further Mordell [9] treated the special case such that $s_{1}=s_{2}=s_{3}$ and evaluated special values at even positive integers in 1958 . 
For $\mathbf{s}=\left(s_{1}, s_{2}, s_{3}\right) \in \mathbb{N}^{3}$, we put

$$
\begin{aligned}
X_{j}^{(l)}=1-x_{j}^{(l)}, & \text { for } j=1,2,3 \text { and } l=2, \ldots, s_{j}, \\
Z_{j}=1-z \prod_{l=2}^{s_{j}} x_{j}^{(l)}, & \text { for } j=1,2,3 .
\end{aligned}
$$

Then the family of polynomials (3.1) for $A_{2}$ can be rewritten as

$$
\mathscr{P}\left(\mathbf{s} ; A_{2}\right)=\left\{X_{j}^{(l)}, Z_{j} \mid j=1,2,3, l=2, \ldots, s_{j}\right\}
$$

and Theorem 3.2 for $A_{2}$ can be described as

Theorem 5.1. For any positive integers $\mathbf{s}=\left(s_{1}, s_{2}, s_{3}\right)$, we have

$$
\begin{aligned}
\mathrm{m}\left(\mathscr{P}\left(\mathbf{s} ; A_{2}\right)\right)= & \frac{(-1)^{S_{3}}}{2^{S_{3}-1}}\left(\zeta_{2}\left(s_{1}, s_{2}, s_{3} ; A_{2}\right)+\zeta_{2}\left(s_{2}, s_{3}, s_{1} ; A_{2}\right)\right. \\
& \left.+\zeta_{2}\left(s_{3}, s_{1}, s_{2} ; A_{2}\right)\right)
\end{aligned}
$$

where $S_{3}=\sum_{j=1}^{3} s_{j}$.

In some case, we can evaluate the right-hand side of (5.2) in terms of Riemann zeta values or multiple zeta values. For instance, Nakamura [10] showed the following formula:

$$
\begin{aligned}
& \zeta_{2}\left(a, b, s ; A_{2}\right)+(-1)^{b} \zeta_{2}\left(b, s, a ; A_{2}\right)+(-1)^{a} \zeta_{2}\left(s, a, b ; A_{2}\right) \\
& =\frac{2}{a ! b !} \sum_{k=0}^{\max (a, b) / 2}\left\{a\left(\begin{array}{c}
b \\
2 k
\end{array}\right)+b\left(\begin{array}{c}
a \\
2 k
\end{array}\right)\right\}(a+b-2 k-1) !(2 k) ! \zeta(2 k) \\
& \quad \times \zeta(a+b+s-2 k),
\end{aligned}
$$

which holds for all $a, b \in \mathbb{N}$ and $s \in \mathbb{C}$ except for the singular points of each side of this formula.

Remark 5.2. The similar formula was proved by Tsumura [15] before Nakamura showed the above formula. However that of Nakamura is simpler than that of Tsumura.

By using the above formula, we have 
Corollary 5.3. For positive even integers $s_{1}$ and $s_{2}$, we have

$$
\begin{aligned}
\mathrm{m}\left(\mathscr{P}\left(\mathbf{s} ; A_{2}\right)\right)= & \frac{(-1)^{S_{3}}}{2^{S_{3}-2} s_{1} ! s_{2} !} \sum_{k=0}^{\max \left(s_{1}, s_{2}\right) / 2}\left\{s_{1}\left(\begin{array}{c}
s_{2} \\
2 k
\end{array}\right)+s_{2}\left(\begin{array}{c}
s_{1} \\
2 k
\end{array}\right)\right\} \\
& \times\left(s_{1}+s_{2}-2 k-1\right) !(2 k) ! \zeta(2 k) \zeta\left(S_{3}-2 k\right) .
\end{aligned}
$$

Example 5.4. (1) $\left(s_{1}, s_{2}, s_{3}\right)=(2,1,1)$,

$$
\begin{aligned}
\mathrm{m}(1-x z, 1-x, 1-z, 1-z) & =\frac{1}{8}\left(2 \zeta_{2}\left(2,1,1 ; A_{2}\right)+\zeta_{2}\left(1,1,2 ; A_{2}\right)\right) \\
& =\frac{3}{8} \zeta(4)=\frac{\pi^{4}}{240} .
\end{aligned}
$$

(2) $\left(s_{1}, s_{2}, s_{3}\right)=(2,2,2)$ (Witten's volume formula)

$$
\begin{aligned}
& \mathrm{m}\left(1-x_{1} z, 1-x_{1}, 1-x_{2} z, 1-x_{2}, 1-x_{3} z, 1-x_{3}\right) \\
& \quad=\frac{3}{32} \zeta_{2}\left(2,2,2 ; A_{2}\right)=\frac{\pi^{6}}{30240} .
\end{aligned}
$$

(3) $\left(s_{1}, s_{2}, s_{3}\right)=(2,2,3)$,

$$
\begin{aligned}
& \mathrm{m}\left(1-x_{1} z, 1-x_{1}, 1-x_{2} z, 1-x_{2}, 1-x_{3}^{(2)} x_{3}^{(3)} z, 1-x_{3}^{(2)}, 1-x_{3}^{(3)}\right) \\
& \quad=-\frac{1}{64}\left(2 \zeta_{2}\left(3,2,2 ; A_{2}\right)+\zeta_{2}\left(2,2,3 ; A_{2}\right)\right)=-\frac{1}{32}(-3 \zeta(7)+2 \zeta(2) \zeta(5)) .
\end{aligned}
$$

\section{2. $B_{2}$ and $C_{2}$ cases}

The multi-variable Witten zeta-function associated with $B_{2}$ and $C_{2}$ is

$$
\zeta_{2}\left(s_{1}, s_{2}, s_{3}, s_{4} ; B_{2}\right)=\sum_{m, n=1}^{\infty} \frac{1}{m^{s_{1}} n^{s_{2}}(m+n)^{s_{3}}(m+2 n)^{s_{4}}} .
$$

Matsumoto [7] first introduced the above function as $\zeta_{\mathfrak{s o}(5)}\left(s_{1}, s_{2}, s_{3}, s_{4}\right)$ and showed the meromorphic continuation of it to the whole $\mathbb{C}^{4}$ space.

For $\mathbf{s}=\left(s_{1}, s_{2}, s_{3}, s_{4}\right) \in \mathbb{N}^{4}$, we put

$$
\begin{aligned}
X_{j}^{(l)} & =1-x_{j}^{(l)}, & & \text { for } j=1,2,3,4 \text { and } l=2, \ldots, s_{j}, \\
Z_{j} & =1-\prod_{i=1}^{2} z_{i}^{\mathcal{C}_{i, j}} \prod_{l=2}^{s_{j}} x_{j}^{(l)}, & & \text { for } j=1,2,3,4,
\end{aligned}
$$


where

$$
\mathcal{C}_{i, j}= \begin{cases}2 & \text { if }(i, j)=(2,2), \\ 0 & \text { if }(i, j)=(2,3),(1,4), \\ 1 & \text { otherwise }\end{cases}
$$

Then the family of polynomials (3.1) for $B_{2}\left(C_{2}\right)$ can be rewritten as

$$
\mathscr{P}\left(\mathbf{s} ; B_{2}\right)=\left\{X_{j}^{(l)}, Z_{j} \mid j=1,2,3,4, l=2, \ldots, s_{j}\right\}
$$

and Theorem 3.2 for $B_{2}\left(C_{2}\right)$ can be described as

Theorem 5.5. For any positive integers $\mathbf{s}=\left(s_{1}, s_{2}, s_{3}, s_{4}\right)$, we have

$$
\begin{aligned}
\mathrm{m}\left(\mathscr{P}\left(\mathbf{s} ; B_{2}\right)\right)= & \frac{(-1)^{S_{4}}}{2^{S_{4}-1}}\left(\zeta_{2}\left(s_{1}, s_{2}, s_{3}, s_{4} ; B_{2}\right)+\zeta_{2}\left(s_{1}, s_{3}, s_{2}, s_{4} ; B_{2}\right)\right. \\
& \left.+\zeta_{2}\left(s_{4}, s_{3}, s_{2}, s_{1} ; B_{2}\right)+\zeta_{2}\left(s_{4}, s_{2}, s_{3}, s_{1} ; B_{2}\right)\right)
\end{aligned}
$$

where $S_{4}=\sum_{j=1}^{4} s_{j}$.

It is known that certain Witten zeta values associated with $B_{2}\left(C_{2}\right)$ can be expressed by using Riemann zeta values. For instance,

Theorem 5.6 Theorem in [14], Proposition 5.2 in [11]. Suppose that $s_{j} \in \mathbb{N} \cup\{0\}$ with $s_{1} \geq 1, s_{1}+s_{2}+s_{3}>1, s_{1}+s_{2}+s_{4}>1, s_{2}+s_{3}+s_{4}>1$, $\sum_{j=1}^{4} s_{j}>2$, and that $\sum_{j=1}^{4} s_{j}$ is odd. Then $\zeta_{2}\left(s_{1}, s_{2}, s_{3}, s_{4} ; B_{2}\right)$ can be expressed as a rational linear combination of products of Riemann zeta values at positive integers.

Therefore $m\left(\mathscr{P}\left(\mathbf{s} ; B_{2}\right)\right)$ with $\sum_{j=1}^{4} s_{j} \equiv 1(\bmod 2)$ can be expressed in terms of Riemann zeta values. Further Nakamura showed an explicit formula which is similar to the right-hand side of (5.4).

Theorem 5.7 Theorem 5.5 in [11]. The following formula holds for $a, b, c \in \mathbb{N}$ and $s \in \mathbb{C}$ except for singular points of each side of the formula:

$$
\begin{array}{r}
\zeta_{2}\left(a, b, s, c ; B_{2}\right)+(-1)^{b} \zeta_{2}\left(c, b, s, a ; B_{2}\right)+(-1)^{a} \zeta_{2}\left(a, s, b, c ; B_{2}\right) \\
+(-1)^{b+c} \zeta_{2}\left(c, s, b, a ; B_{2}\right)=2 \sum_{d=0}^{a}\left(\begin{array}{c}
a+b-d-1 \\
a-d
\end{array}\right) \sum_{j=0}^{\max (c, d) / 2}
\end{array}
$$




$$
\begin{aligned}
& \times\left\{\left(\begin{array}{c}
c+d-2 j-1 \\
c-2 j
\end{array}\right)+\left(\begin{array}{c}
c+d-2 j-1 \\
d-2 j
\end{array}\right)\right\} 2^{2 j-c-d} \zeta(2 j) \\
& \times \zeta(a+b+c+s-2 j)+2 \sum_{d=0}^{b}(-1)^{d}\left(\begin{array}{c}
a+b-d-1 \\
b-d
\end{array}\right) \sum_{j=0}^{\max (c, d) / 2} \\
& \times\left\{\left(\begin{array}{c}
c+d-2 j-1 \\
c-2 j
\end{array}\right)+\left(\begin{array}{c}
c+d-2 j-1 \\
d-2 j
\end{array}\right)\right\} \zeta(2 j) \zeta(a+b+c+s-2 j) .
\end{aligned}
$$

By using Theorem 5.7, we can concretely evaluate the right-hand side of (5.4) in some cases.

Example 5.8. $\quad(1)\left(s_{1}, s_{2}, s_{3}, s_{4}\right)=(1,2,1,1)$

$$
\begin{aligned}
\mathrm{m} & \left(1-z_{1} z_{2}, 1-x_{2} z_{1} z_{2}^{2}, 1-x_{2}, 1-z_{1}, 1-z_{2}\right) \\
& =-\frac{1}{2^{4}}\left\{2 \zeta_{2}\left(1,2,1,1 ; B_{2}\right)+2 \zeta_{2}\left(1,1,2,1 ; B_{2}\right)\right\} \\
& =-\frac{1}{2^{4}}\left\{-3 \zeta(5)+\frac{\pi^{2}}{3} \zeta(3)\right\},
\end{aligned}
$$

since we see that

$$
\zeta_{2}\left(1,2,1,1 ; B_{2}\right)=-\frac{13}{8} \zeta(5)+\frac{\pi^{2}}{6} \zeta(3), \quad \zeta_{2}\left(1,1,2,1 ; B_{2}\right)=\frac{1}{8} \zeta(5)
$$

from the list in [14, p. 151].

(2) $\left(s_{1}, s_{2}, s_{3}, s_{4}\right)=(2,2,2,2)$ (Witten's volume formula)

$$
\begin{aligned}
& \mathrm{m}\left(1-x_{1} z_{1} z_{2}, 1-x_{2} z_{1} z_{2}^{2}, 1-x_{3} z_{1}, 1-x_{4} z_{2}, 1-x_{1}, 1-x_{2}, 1-x_{3}, 1-x_{4}\right) \\
& =\frac{\zeta_{2}\left(2,2,2,2 ; B_{2}\right)}{2^{5}}=\frac{\pi^{8}}{9676800}, \\
& \quad \text { since } \zeta_{2}\left(2,2,2,2 ; B_{2}\right)=\pi^{8} / 302400(\text { see }[4,(264)]) .
\end{aligned}
$$

\section{3. $G_{2}$ case}

The multi-variable Witten zeta-function associated with $G_{2}$ is

$$
\begin{aligned}
\zeta_{2} & \left(s_{1}, s_{2}, s_{3}, s_{4}, s_{5}, s_{6} ; G_{2}\right) \\
& =\sum_{m, n=1}^{\infty} \frac{1}{m^{s_{1}} n^{s_{2}}(m+n)^{s_{3}}(m+2 n)^{s_{4}}(m+3 n)^{s_{5}}(2 m+3 n)^{s_{6}}} .
\end{aligned}
$$


For $\mathbf{s}=\left(s_{1}, \ldots, s_{6}\right) \in \mathbb{N}^{6}$, we put

$$
\begin{aligned}
X_{j}^{(l)} & =1-x_{j}^{(l)}, & & \text { for } j=1, \ldots, 6 \text { and } l=2, \ldots, s_{j}, \\
Z_{j} & =1-\prod_{i=1}^{4} z_{i}^{\mathcal{C}_{i, j}} \prod_{l=2}^{s_{j}} x_{j}^{(l)}, & & \text { for } j=1, \ldots, 6,
\end{aligned}
$$

where

$$
\mathcal{C}_{i, j}= \begin{cases}3 & \text { if }(i, j)=(3,2),(4,2) \\ 2 & \text { if }(i, j)=(2,2),(4,1) \\ 1 & \text { if }(i, j)=(1,1),(1,2),(2,1),(3,1) \\ 0 & \text { otherwise }\end{cases}
$$

Then the family of polynomials (3.1) for $G_{2}$ can be rewritten as

$$
\mathscr{P}\left(\mathbf{s} ; G_{2}\right)=\left\{X_{j}^{(l)}, Z_{j} \mid j=1, \ldots, 6, l=2, \ldots, s_{j}\right\}
$$

and Theorem 3.2 for $G_{2}$ can be described as

Theorem 5.9. For any positive integers $\mathbf{s}=\left(s_{1}, \ldots, s_{6}\right)$, we have

$$
\begin{aligned}
\mathrm{m}\left(\mathscr{P}\left(\mathbf{s} ; G_{2}\right)\right)= & \frac{(-1)^{S_{6}}}{2^{S_{6}-1}}\left\{\zeta_{2}\left(s_{1}, s_{2}, s_{3}, s_{4}, s_{5}, s_{6} ; G_{2}\right)\right. \\
& +\zeta_{2}\left(s_{6}, s_{3}, s_{4}, s_{2}, s_{1}, s_{5} ; G_{2}\right)+\zeta_{2}\left(s_{6}, s_{4}, s_{3}, s_{2}, s_{5}, s_{1} ; G_{2}\right) \\
& +\zeta_{2}\left(s_{5}, s_{4}, s_{2}, s_{3}, s_{6}, s_{1} ; G_{2}\right)+\zeta_{2}\left(s_{5}, s_{2}, s_{4}, s_{3}, s_{1}, s_{6} ; G_{2}\right) \\
& \left.+\zeta_{2}\left(s_{1}, s_{3}, s_{2}, s_{4}, s_{6}, s_{5} ; G_{2}\right)\right\} .
\end{aligned}
$$

In [5], Komori et al. considered a "Weyl group symmetric" linear combination of the Witten zeta-function associated with $G_{2}$ which is similar to the right-hand side of (5.6) and evaluated special values at positive integers of such function (see Section 2 in [5]). From their theorem, we have

Corollary 5.10. For even positive integers $\left(s_{1}, \ldots, s_{6}\right)$, we have

$$
\mathrm{m}\left(\mathscr{P}\left(\mathbf{s} ; G_{2}\right)\right)=\frac{(-1)^{S_{6}}}{2^{S_{6}-1}}\left(\prod_{\alpha \in \Delta_{+}\left(G_{2}\right)} \frac{(2 \pi i)^{s_{\alpha}}}{s_{\alpha} !}\right) P\left(\mathbf{s} ; 0 ; G_{2}\right),
$$

where $P\left(\mathbf{s} ; 0 ; G_{2}\right)$ are certain rational numbers depend on $\mathbf{s}$ and the root system $G_{2}$. 
Remark 5.11. Numbers $P\left(\mathbf{s} ; 0 ; G_{2}\right)$ can be calculated. Indeed, $P\left(\mathbf{s} ; 0 ; G_{2}\right)$ are defined as coefficients of the Taylor expansion of a function $F\left(\mathbf{t} ; \mathbf{y} ; G_{2}\right)$ introduced in [5].

\section{Acknowledgements}

The author would like to express his sincere gratitude to Professors Yasushi Komori, Takashi Nakamura and Kazuhiro Onodera for their various advice and helpful suggestion. Thanks are also due to Professor Yasuo Ohno for his comments and encouragement. This work was supported by Grants-in-Aid for Young Scientists (B) no. 23740036.

\section{References}

[1] P.E. Gunnells and R. Sczech, Evaluation of Dedekind sums, Eisenstein cocycles, and special values of L-functions, Duke Math. J. 118 (2003), 229-260.

[2] Y. Komori, K. Matsumoto and H. Tsumura, Zeta functions of root systems, in "The Conference on L-Functions", eds. L. Weng and M. Kaneko, World Scientific Publishing, 2007, 115-140.

[3] Y. Komori, K. Matsumoto and H. Tsumura, On Witten multiple zetafunctions associated with semisimple Lie algebras II, J. Math. Soc. Japan, 62 (2010), 355-394.

[4] Y. Komori, K. Matsumoto and H. Tsumura, On Witten multiple zetafunctions associated with semisimple Lie algebras III, in "Multiple Dirichlet Series, $L$-functions and Automorphic Forms", eds. D. Bump et al., Progr. Math., 300, Birkhäuser, Basel, 2012, 223-286.

[5] Y. Komori, K. Matsumoto and H. Tsumura, On Witten multiple zetafunctions associated with semisimple Lie algebras IV, Glasg. Math. J. 53 (2011), 185-206.

[6] N. Kurokawa, M. Lalín and H. Ochiai, Higher Mahler measures and zeta functions, Acta Arith. 135 (2008), 269-297.

[7] K. Matsumoto, On Mordell-Tornheim and other multiple zetafunctions, Proceedings of the Session in analytic number theory and Diophantine equations (Bonn, January-June 2002), eds. D. R. HeathBrown and B. Z. Moroz, Bonner Mathematische Schriften Nr. 360, Bonn 2003, no. 25, 17. 
[8] K. Matsumoto and H. Tsumura, On Witten multiple zeta-functions associated with semisimple Lie algebras I, Ann. Inst. Fourier (Grenoble) 56 (2006), 1457-1504.

[9] L.J. Mordell, On the evaluation of some multiple series, J. London Math. Soc. 33 (1958), 368-371.

[10] T. Nakamura, A functional relation for the Tornheim double zeta function, Acta Arith. 125 (2006), 257-263.

[11] T. Nakamura, Double Lerch value relations and functional relations for Witten zeta functions, Tokyo J. Math. 31 (2008), 551-574.

[12] M.V. Subbarao and R. Sitaramachandrarao, On some infinite series of L. J. Mordell and their analogues, Pacific J. Math. 119 (1985), 245-255.

[13] L. Tornheim, Harmonic double series, Amer. J. Math. 72 (1950), $303-314$

[14] H. Tsumura, On Witten's type of zeta values attached to $S O(5)$, Arch. Math. (Basel) 82 (2004), 147-152.

[15] H. Tsumura, On functional relations between the Mordell-Tornheim double zeta functions and the Riemann zeta function, Math. Proc. Cambridge Phil. Soc. 142 (2007), 395-405.

[16] D. Zagier, Values of zeta functions and their applications, in First European Congress of Math., Paris, Vol. II, Prog. Math., 120, Birkhäuser, Basel, 1994, 497-512.

[17] E. Witten, On quantum gauge theories in two dimensions, Comm. Math. Phys. 141 (1991), 153-209.

Osaka University of Health and Sport Sciences,

Asashirodai 1-1

KUMATORI-CHO

SENNAN-GUN OSAKA 590-0496

JAPAN

E-mail address: ysasaki@ouhs.ac.jp

Received September 5, 2012 